\section{THE DEVELOPMENT OF MECHANISM BASED ON HYBRID KINEMATIC STRUCTURE}

V. Poppeova, J. Uricek, VI. Bulej

Department of Automation and Production Systems

Faculty of Mechanical Engineering, University of Zilina Zilina, Slovak Republic

e-mail: viera.poppeova@fstroj.uniza.sk

Initial enthusiasm for the parallel kinematics in the eighties of the last century slumped significantly during the last twenty years. There were found some disadvantages of standard parallel kinematic structures. The experts around the world began to seek new solutions of outlined problems. They defined several possible ideas which could eliminate the drawbacks and improve the properties and advantages of parallel machines. One of these ways was also the application of mechanisms based on the kinematic structure that consisted of serial and parallel substructures as well. This article is focused on the development of mechanism with hybrid kinematic structure called Trivariant. This mechanism is similar to the well-known and succesful concept called Tricept, represented by various machines.

Keywords parallel kinematic structure, hybrid kinematic structure, robot, Trivariant, control

\section{Introduction}

The main mechanisms with parallel architecture have the end-effector (platform) connected to the frame (base) through a number of kinematic chains (legs). Stewart presented his platform in 1965 Since then, several authors have proposed a large variety of designs (until today there were designed about 200 different architectures of PKS) [Merlet 2000]. Hexapod represents the best-known fully parallel manipulator with 6 DOF which ensure positioning and also orientation of the end-effector. However, despite intensive efforts by both research and industry, the commercial success of hexapod has still been limited.

Initial enthusiasm for the parallel kinematics in the eighties of the last century slumped significantly during the last twenty years. When the parallel kinematic structure (PKS) was first proposed for machine tools, there were many generic claims made regarding its advantages. Among these claims there were assertions of "an almost infinite rigidity because the structural elements are not subject to any bending", improved dynamic performance and better accuracy. These generic claims are now being challenged, and some have been found to be false [Tlusty 1999].

The parameters of real devices didn't achieve the expectations of engineers and the machines based on PKS didn't reach the predicted stiffness and accuracy as well as the workspace was significantly limited by singular positions.

\section{Most important disadvantages of standard PKS}

[Merlet 2000, Valasek 2009]:

- singular positions and singular configurations within the workspace (then reduction of workspace);

- heterogeneous distribution of properties along the workspace,

- application of joints with point contac

(lower stiffness of whole mechanism);

- floating mount of drives.

\section{New solutions for the field of PKS application}

The experts around the world have begun to seek new solutions of outlined problems. They have defined several possible ideas which can eliminate these drawbacks and improve the properties and advantages of parallel kinematic machines (PKM). For example we can see that there is a growing tendency to focus on the parallel mechanisms with 3 translational joints (3 DOF) [Tlusty 1999, Valasek 2009] instead of 6 DOF fully parallel mechanisms.

During the study of the literature and other various sources we have met with a number of tendencies. As the most important and most common tendencies we can consider:

- redundancy (stiffness, accuracy, mobility, high dynamics and long movement);

- application of hybrid kinematic structures (tricept, trivariant, delta robot with orientation);

- reconfigurable kinematic structures;

- compensation of tool deflection, torsional vibrations, static, dynamic and thermal errors;

- replacement of spherical joints (point contact) by revolute joints (linear contact);

- wide application of mechatronical components - piezoelectric compensator, active electromagnetic guiding system, unit for micropositioning;

- application of special components (cardan joint with wide range and electromagnetic ball joint);

- application of special materials such as carbon fiber and aluminum foam;

- application of PKS for the design of measuring systems [Benes 2009];

- designing of multi-tasking machines - an integration of machining and manipulation.

\section{Mechanisms with hybrid kinematic structure}

When the standard serial and also the parallel mechanisms achieve their limits we can use the mechanisms based on hybrid kinematic structure, which combine the advantages both types of kinematics. During the last decade, the well-known mechanism with hybrid kinematic structure called Tricept (Figure $1-a$ ), has found various commercial applications such as high-speed milling, welding and component assembling in an aeronautical and automotive industry [Neumann 2002]. Another example of concept based on HKS is also the mechanism called Trivariant, which is the main topic of this article. We have met with this mechanism first time in the article written by Meng [2005]. As an example of the Trivariant real application is the mechanism Trivariant B-2000 produced by the Jiangyin Naeriie Robotics Company, Ltd. (cooperated with Tianjin University) located in China.

The multifunctional robotic device Trivariant B-2000 (figure $1-$ b) is intended for automation of welding, machining, assembly operations and "pick-and-place" handling. Position accuracy is lower than accuracy of Tricept, but it has a relatively large workspace (1 $500 \times 1500$

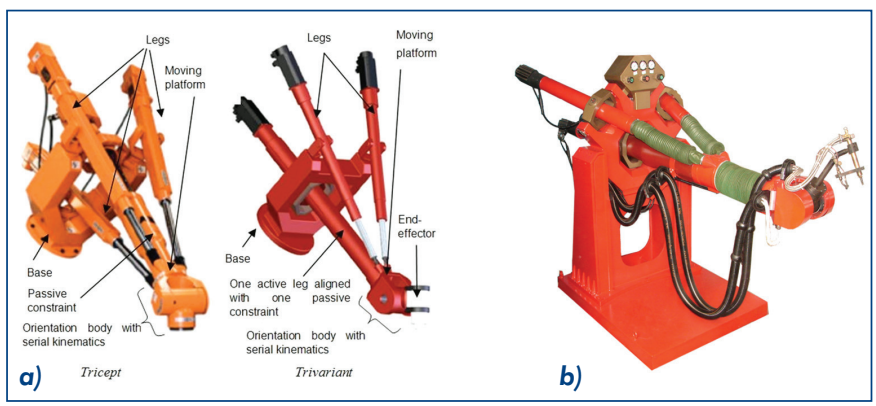

Figure 1. Machines with hybrid kinematic structure a-difference between the Tricept and Trivariant, $b$ - multifunctional robot Trivariant B-2000 made by Jiangyin Naeriie Robotics Company, Ltd. [Jiangyin 2010] 
$x 1400 \mathrm{~mm}$ ). This mechanism can reach the speed up to $0,8 \mathrm{~m} \cdot \mathrm{s}^{-1}$ and acceleration up to $5 \mathrm{~m} \cdot \mathrm{s}^{-2}$ [Jiangyin 2010].

Authors of this article have decided to design and build the mechanism Trivariant such as the appropriate software for the simulation and control system in their department.

\section{Design of Trivariant functional model}

The DOF number and the motion type of all parallel mechanisms depend on the type and on the architecture of the kinematic structure which consists of apportionable parts connected together. Generally, it is possible to choose from these four basic types of joints (ordered by increasing DOF): revolute joint $(R)-1$ rotational DOF, prismatic joint $(P)-1$ translational DOF, universal joint $(U)-2$ rotational DOF, spherical joint (S) - 3 rotational DOF [Knoflicek 2006]. Thereafter, the joints are connected together by links and actuated by linear or rotational actuators. The design of the architecture is very important because it will determine the stiffness and the workspace dimensions [Neugebauer 2002]. In our case, it is necessary to obtain 5 DOF and a good ratio between the dimensions of both the mechanism and the workspace.

\subsection{Kinematic scheme}

From the viewpoint of mechanisms, Trivariant (figure 2) can be decomposed into one spherical-co-ordinate parallel mechanism (PM) and a serial extension ( $\mathrm{SE}$; or also serial module) based on two rotational joints with orthogonal axes. The subsystem with parallel kinematics represents the positioning of the end-effector in mechanism workspace whilst the serial extension covers its orientation. The architecture of the mechanism is very similar to the classical Tricept The difference lies in PM, where there is one active leg aligned with one passive leg.

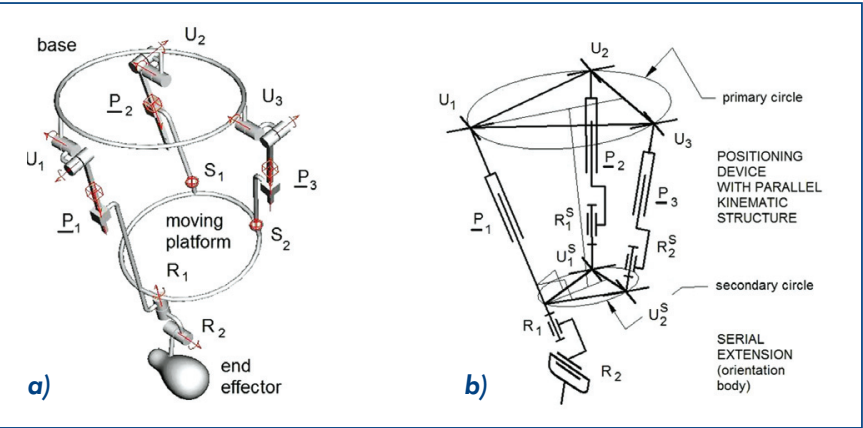

Figure 2. Original (a) and modified (b) kinematic scheme of Trivariant [Poppeova 2009]

In more detailed view, the PM consists of a fixed base, a moving platform and three active legs. Two of them are identical and based on architecture UPS. The last one is different and based on architecture UP which means that the moving platform is direct connected to this leg (rigid connection). This leg allows only two rotationa and one translational movement of the moving platform. It means that one platform's rotation around the $\mathrm{P}$ joint axis and two translations perpendicular to the $\mathrm{P}$ joint are disabled. The symbols $\mathrm{U}, \mathrm{P}$ and $S$ represent the universal, prismatic and spherical joint. Additionally, $\underline{P}$ denotes an active prismatic joint usually powered by a servomotor The $\mathrm{S}$ joint at the end of each two identical legs UPS can be replaced by one $R$ and one $U$ joint - it means the architecture of the legs was changed to UP (RU). In spite of that fact, the number of DOF is still 3 (figure 2 -b).

\subsection{Workspace of Trivariant functional model}

One of the most important properties of all devices for machining or manipulation is the shape and the size of their workspace. Forward kinematics can be used for its determination and evaluation. The workspace was created by using boundary positions of each part of the kinematic structure.

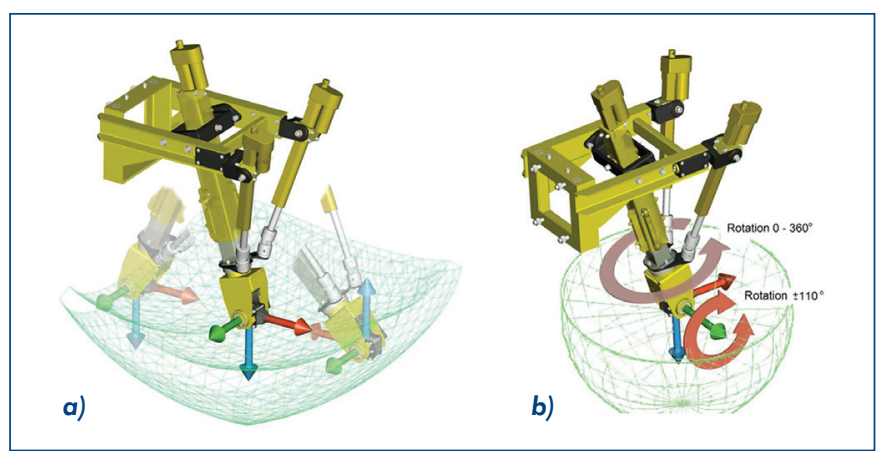

Figure 3. The Workspace (a) of Trivariant designed at University of Žilina and its end-effector mobility range for orientation (b)

Serial extension does for the end-effector orientation. It also compensates the angular displacement of moving platform during its translation. SE with 2 DOF can rotate around two axes (see figure $3-b)$ - the first one in an angular range from 0 to $360^{\circ}$ and the second one in range $\pm 110^{\circ}$. Thanks to these tilt angles mechanism can do machining from 5 sides and $5 \mathrm{D}$ machining too. It is also possible to apply it as a robot device for handling operations but in this case one more rotational axis has to be added into the kinematic scheme of serial extension. Thereby, we obtain a mechanism with 6 DOF.

In figure 3 and 4 is shown the result of Trivariant workspace visualization. Trivariant has a slightly different kinematic structure compare to Tricept. Therefore the workspace is different too. It is possible to reach a bit higher mobility range by using Trivariant. That can be considered as one of the advantages of this kinematic structure. In figure 4 is also shown one example of workpiece size (orange dot-line rectangles). Its dimensions are approximately 380 x $300 \times 150 \mathrm{~mm}$ by actuators maximal extensions up to $250 \mathrm{~mm}$.

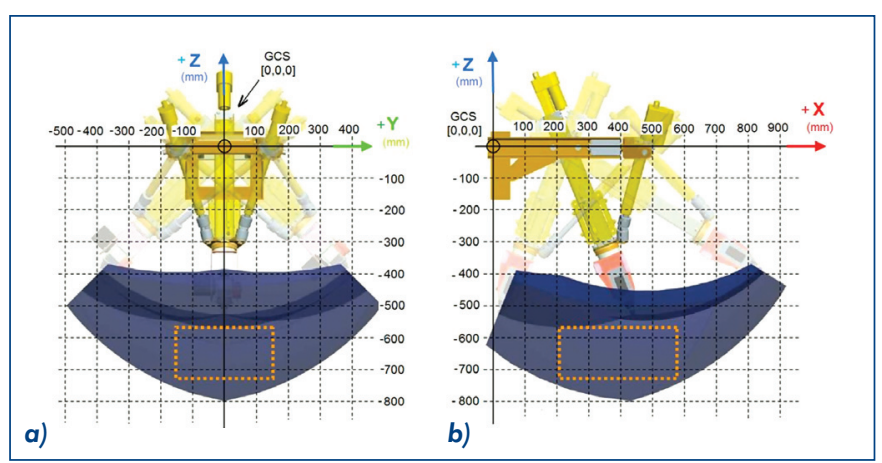

Figure 4. Trivariant workspace - front (a) and side view (b)

\subsection{Singular configuration within the workspace}

During the solution of the mathematical model for Trivariant was also necessary to treat with encountered singular positions, that is to

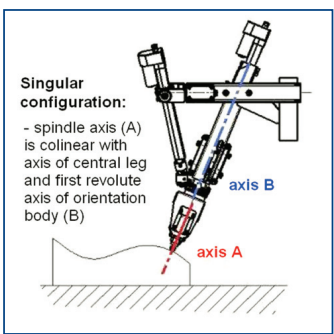

Figure 5. Singular configuration located within the Trivariant workspace [Bulej 2010] 
say, those positions where the mechanism loses one or more DOF. We were focused only to deal with singular points lying within the workspace of mechanism. It is also remarkable that one singular position could be located at any point within the workspace. This singular position occurs in a specific configuration of the mechanism (figure 5), especially when the axis of the end-effector (for example axis of milling spindle) is collinear with the first rotational axis of serial extension SE. In this case, it is possible to reach the position independently of the angular displacement of the first rotational joint of SE. For this situation, we added a special condition into the mathematical model of the mechanism which determines its behaviour in this precise case.

\section{Control system}

Simulation software was created (in programming language Delphi) for off-line programming and simulation of Trivariant movement as well as for its direct control, 3D visualization and carrying out some computer analyses.

During the simulation software uses the mathematical model and calculates all acting variables corresponded to required trajectory. In the next section there is described the method how to get the mathematical model of Trivariant prototype. All the calculations are performed in the Cartesian co-ordinate system. One or more co-ordinate systems are rigid connected to each part of the mechanism. Co-ordinate system of the frame is considered as the global coordinate system (GSS). To determine the relative position and orientation between the separate parts of kinematic structure there are used homogeneous coordinates and homogeneous transformations.

\subsection{Trivariant mathematical model and inverse kinematics}

The mathematical model of Trivariant represents a description of a real mechanism by system of mathematical equations. In order to control the movement of a programmed trajectory, it is necessary to know the functional relations between a tool centre point (TCP) and active variable of each drive. Therefore there was created an inverse kinematics for this machine. Inverse kinematics was solved by homogenous matrix transformations. We can write the general form of transformation matrix for spatial case because of the mechanism carries out general spatial movement.

General form of transformation matrix [Durica 2009]:

$$
H_{1}^{0}=\left(\begin{array}{cccc}
n_{x} & o_{x} & a_{x} & p_{x} \\
n_{y} & o_{y} & a_{y} & p_{y} \\
n_{z} & o_{z} & a_{z} & p_{z} \\
0 & 0 & 0 & 1
\end{array}\right)=\left(\begin{array}{cccc}
n & o & a & p \\
0 & 0 & 0 & 1
\end{array}\right)=\left(\begin{array}{cc}
R_{3 x 3} & T_{3 x 1} \\
f_{1 \times 3} & s_{1, x 1}
\end{array}\right)
$$

\section{where}

$\mathrm{n}=\left(\mathrm{n}_{\mathrm{x}}, \mathrm{n}_{\mathrm{y}}, \mathrm{n}_{\mathrm{z}}\right)^{\top}$ - directional vector on $X$-axis,

$\mathrm{o}=\left(\mathrm{o}_{x}, \mathrm{o}_{y}, \mathrm{o}_{z}\right)^{\top}$ - directional vector on $\mathrm{Y}$-axis,

$a=\left(a_{x}, a_{y}, a_{z}\right)^{\top}$ - directional vector on Z-axis,

$p=\left(p_{x}, p_{y}, p_{z}\right)^{\top}$ - directional vector between two co-ordinate systems,

$R_{3 \times 3} \quad-$ rotation matrix,

$\mathrm{T}_{3 \times 1}^{3 \times 3} \quad$ - translation matrix,

$f_{1 \times 3}^{3 \times 1} \quad-$ perspective matrix,

$\mathrm{s}_{1 \times 1} \quad-$ scale matrix

Then we can obtain the position and orientation of the end-element by $\mathrm{n}-1$ transformations (transformations of co-ordinate systems corresponded to each mechanism element). The transformations are calculated in the same order as these elements are located in solved kinematic loops.
Final mechanism matrix is represented by the equation

$$
H_{n}^{0}=M_{1} \cdot \ldots \cdot M_{n}
$$

where $M_{1}$ and $M_{n}$ represent the $1^{\text {st }}$ or the $n^{\text {th }}$ transformation of co-ordinate system (translation or rotation).

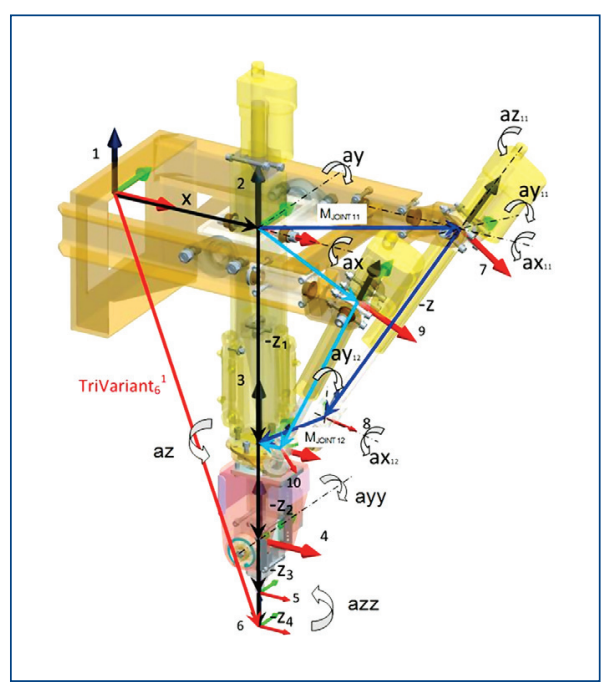

Figure 6. Kinematic scheme of Trivariant with coordinate systems (CS), movements and three kinematic loops [Poppeova 2009]. 1 - frame (GCS), 2 - central U joint, 3 - moving platform, 4 - basic part of serial extension, 5 - end-effector, 6-tool, 7 - top-left $U$ joint, 8 - bottom-left $U$ joint, 9 - top-right $U$ joint, 8 - bottom-right $U$ joint, $M_{\text {JOINT 11 }}$ - linear displacement of top-left $U$ joint, $M_{\text {JOINT 12 }}$ - linear displacement of bottom-left $U$ joint.

At the beginning, the kinematic scheme of Trivariant is decomposed into three separate loops (figure 6), which can be used for calculation of the whole mechanism. These loops we describe are composed by three basic matrixes (each matrix for one loop) [Durica 2009]. The solution of the main loop (black arrows and the red one) transformation matrix gives us the inverse kinematics of main loop. By analogous way we obtain the inverse task of kinematics for two remaining loops.

First loop description:

$$
\begin{aligned}
\operatorname{Trivariant}_{6}^{1}= & \operatorname{Trans}(x, 0,0) \cdot \operatorname{Rot} Y(a y) \cdot \operatorname{Rot} X(a x) \cdot \operatorname{Trans}\left(0,0,-z_{1}\right) \cdot \operatorname{Rot} Z(a z) . \\
& \cdot \operatorname{Trans}\left(0,0,-z_{2}\right) \cdot \operatorname{Rot} Y(a y y) \cdot \operatorname{Trans}\left(0,0,-z_{3},-z_{4}\right) \cdot \operatorname{Rot} Z(a z z)
\end{aligned}
$$

Second loop description - actuator Nr. 1:

$$
\begin{array}{r}
\operatorname{Rot} Y(a y) \cdot \operatorname{Rot} X(a x) \cdot \operatorname{Trans}\left(0,0,-z_{1}\right)=M_{\text {JoINT11 }} \cdot \operatorname{Rot} X(a x 11) \cdot \operatorname{Rot} Y(a y 11) \cdot \\
\cdot \operatorname{Trans}(0,0,-z) \cdot \operatorname{Rot} Z(a z 1)) \cdot \operatorname{Rot}(a y 12) \cdot \operatorname{Rot} X(a x 12) \cdot M_{\text {JonNT12 }}
\end{array}
$$

Third loop description - actuator Nr. 2:

$$
\begin{aligned}
& \operatorname{Rot} Y(a y) \cdot \operatorname{Rot} X(a x) \cdot \operatorname{Trans}\left(0,0,-z_{1}\right)=M_{\text {JoINT21 }} \cdot \operatorname{RotX}(a x 21) \cdot \operatorname{RotY}(a y 21) . \\
& \cdot \operatorname{Trans}(0,0,-z) \cdot \operatorname{Rot} Z(a z 21) \cdot \operatorname{Rot} Y(a y 22) \cdot \operatorname{Rot} X(a x 22) \cdot M_{\text {JOINT } 22}
\end{aligned}
$$

Inverse kinematics computes the joint variables corresponding to locations of the machine end-effector. It provides to the machine tool a relative movement between the tool and the workpiece by trajectories, which are necessary to create a part with required shape, quality and with maximum repeatability. For machining, linear, circular interpolation and their composition are used to get a final tool path. 


\subsection{Control system architecture and communication}

Whereas simulation software works on non-deterministic operating system (OS) it was necessary to design a special communication board to ensure the deterministic control process. Data between personal computer (PC) and motor drivers (MD) are routed by communication board with serial communication (TX, RX). Data received from simulation software are stored in floating round buffer, which is located in the communication board. Then they are periodically routed into the corresponding motor driver. Whereas the communication between PC and the communication board are buffered it is used a RTS/CTS flow control of serial communication. After receiving data from $\mathrm{PC}$, communication board selects corresponding motor driver (MD) by the links "Select MD". Then, the selected MD enables serial communication and receiving data. The real position of drive is sent to $\mathrm{PC}$ at the same time. This control system (figure 7) is designed for machines with up to 6 drives.

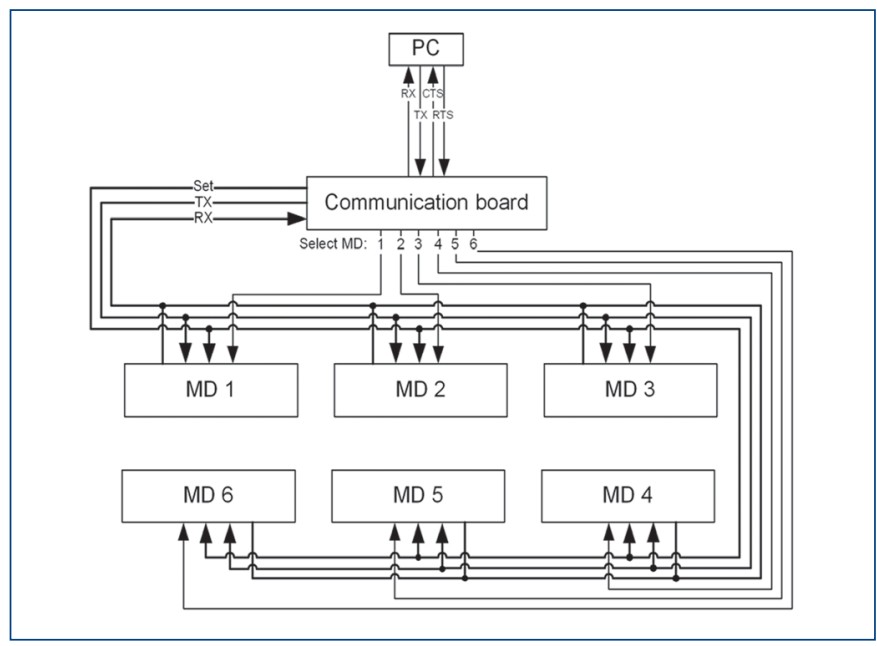

Figure 7. Architecture of control system [Durica 2009]

One optical incremental sensor was mounted in each drive unit (linear or rotational actuator). The signals obtained from these incremental sensors are used to calculate the real position (linear or angular displacements) of each actuator.

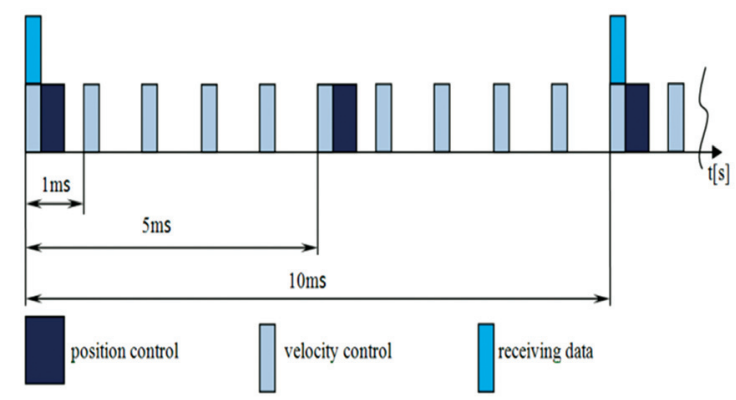

Figure 8. Time diagram of motor driver working

According to the sensors type there was defined 10 ms working period of the whole control system. Furthermore it was necessary to have $5 \mathrm{~ms}$ period for the position control and $1 \mathrm{~ms}$ period for the velocity control to reach the continuous movements without jerks (see figure 8). These periods are enough for appropriate response of whole control loop. The control algorithms for motor drivers was designed and verified in Matlab/Simulink. The controller (figure 9) with cascade arrangement of position and velocity control was designed. The first loop is used for the position control (P-controller) and the second one for the velocity control (PI-controller) [Durica 2009].

Position controller compares the required position with feedback. It shifts the potential action to velocity controller. Velocity controller compares the actual value with the velocity feedback. The controller is designed to reach the required position with minimum overshoot. Each drive was supplemented by end sensor which was used for synchronization.

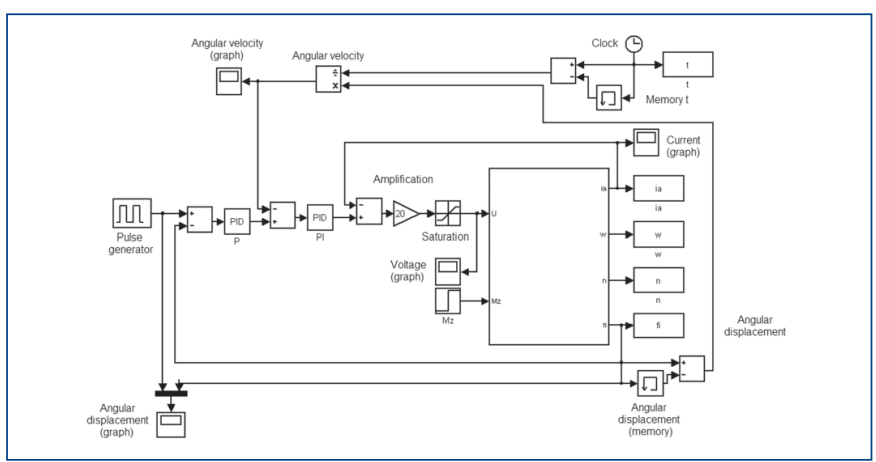

Figure 9. Model of drive cascade control simulated in Matlab/Simulink [Durica 2009]

\section{Conclusion}

The mechanisms with parallel and hybrid kinematic structure in the design of machine tools and robots allow the idea of HSC technologies. As a result, a noteworthy progress in the field of parallel and hybrid mechanisms was carried out during the last few years. In spite of that fact, there are still many problems such as friction in joints, singularities, calibrations, etc. The machines based on hybrid kinematic structures are shown as very suitable solution and alternative for machines with serial kinematics in robotics and also in machining tasks. One of these mechanisms is the Trivariant.

A small-scale Trivariant functional model was built for testing, functional verification, education and training at the University of Zilina. The mechanical subsystem, the simulation software and the control system based on standard PC were designed especially for this prototype. The software designed for simulation and control of Trivariant prototype allows two working modes - as a machine tool with $5 \mathrm{DOF}$ as well as a robot with $6 \mathrm{DOF}$. The designed algorithm interpolates a tool path and in each point of the trajectory calculates all the required parameters for machine control and for visualization by using proposed inverse kinematic. The trajectory can be controlled manually or automatically during the simulation. CL-Data generated by CAM system (in our case Pro/ENGINEER) are used for trajectory planning in automatic mode. Thanks to the workspace of the Trivariant prototype the end-effector can move within and also outside of a block with the dimensions approximately $380 \times 300 \times 150 \mathrm{~mm}$. The functionality of the simulation software and the control system were verified upon the functional model at our department. Defined target to develop the hybrid kinematic structure can be considered fulfilled. We want to improve the properties of mechanical subsystem and also carry out some experiments to the future.

\section{Acknowledgement}

This article was made under the support of Grant Agency KEGA Slovak Republic - project No: 086 - 051 ŽU.

\section{References}

[Benes 2009] BENES, P., VALASEK, M., SVEDA, J. 2009. Measurement and calibration of machine tools in 6 DOFs in large workspace. In: Journal of Machine Engineering. ISSN 1895-7595, 2009, vol. 9, no. 3, p. 77-87. 
[Bulej 2010] BULEJ, V: The Development of Mechanism with hybrid kinematic structure. Disertation thesis, University of Žilina, Faculty of Mechanical Engineering, Slovakia, 2010. (in Slovak)

[Durica 2009] DURICA, J: The Development of Control System for PKS (in Slovak). Disertation thesis, University of Zilina, Faculty of Mechanical Engineering, Slovakia, 2009.

[Jiangyin 2010] Jiangyin Nareaije Robot Co., Ltd. - The Multifunctional Trivariant B-2000 Robot. 2010. [online]. 2010. [cit. 201004-14]. Available on internet: <http://www.chinanej.com/en/ ProductShow.asp? |D $=215>$.

[Knoflicek 2006] KNOFLICEK R., PLSEK L.: PKS for Production Machines and Industrial Robots. VUT Brno, 2006 (in Czech)

[Meng 2005] MENG, L., et al: Dynamic Formulation and Performance Comparision of the 3-DOF Modules of Two Reconfigurable PKM - the Tricept and the TriVariant. In: Journal of Mechanical Design. ISSN: 1050-0472, november 2005, vol. 127, isssue 6, p. 1129-1136.

[Merlet 2000] MERLET, J.-P.: Parallel robots. Kluwer Academic Publisher, Dordrecht, 2000, p. 327, ISBN 0-7923-6308-6

[Neugebauer 2002] NEUGEBAUER R., HARZBECKER C., et al: Parallel Kinematic Structures in Manufacturing. In: Development Methods and Application Experience of Parallel Kinematics. Fraunhofer Institute for Machine Tools and Forming Technology IWU, Chemnitz, Germany, 2002, p. 17-47, pp.1025, ISBN: 3-928921-76-2.
[Neumann 2002] NEUMANN K. E: Tricept Applications. In: Development Methods and Application Experience of Parallel Kinematics. Fraunhofer Institute for Machine Tools and Forming Technology IWU, Chemnitz, Germany, 2002, p. 547-551, pp.1025, ISBN: 3-928921-76-2.

[Poppeova 2009] POPPEOVA, V. et al: The Development of Mechanism with Hybrid Kinematic Structure Prototype. In Journal of Machine Engineering. ISSN 1895-7595, 2009, vol. 9, No. 3, p. 102-109.

[Tlusty 1999] TLUSTY, J. - et al.: Fundamental Comparison of the Use of Serial and Parallel Kinematics for Machines Tools. In: Annals of the CIRP, Vol.48, No.7, 1999, p.351-356, ISSN 0007-8506

[Valasek 2009] VALASEK, M: Actual situation in the development of the hybrid kinematic structure. In: Trends in the R\&D of the machine tools, April 2009, CVUT Praha, ISBN: 978-80-9040771-8. (In Czech)

\section{Contacts:}

Assoc. Prof. Viera Poppeova,

Department of Automation and Production Systems, Faculty of Mechanical Engineering, University of Zilina 01026 Zilina, Slovak Republic

tel.: 00421415132809

e-mail: viera.poppeova@fstroj.uniza.sk 